\title{
REFORMULAÇÃO PARA UM PROBLEMA INTEGRADO DE DIMENSIONAMENTO E SEQUENCIAMENTO DE LOTES
}

\author{
Eduardo Delcides Bernardes \\ Silvio Alexandre de Araujo \\ Socorro Rangel* \\ Dep. de Ciências da Computação e Estatística \\ Instituto de Biociências, Letras e Ciências Exatas \\ UNESP-Univ. Estadual Paulista \\ São José do Rio Preto - SP \\ eduardodbernardes@gmail.com \\ saraujo@ibilce.unesp.br \\ socorro@ibilce.unesp.br \\ * Corresponding author / autor para quem as correspondências devem ser encaminhadas \\ Recebido em 11/2008; aceito em 02/2010 após 2 revisões \\ Received November 2008; accepted February 2010 after two revisions
}

\section{Resumo}

Neste trabalho, são apresentadas algumas novas restrições e uma formulação estendida para um Modelo Integrado de Dimensionamento e Sequenciamento de Lotes da literatura. Considera-se um processo produtivo em que é necessária a produção de determinado material que é transformado em itens finais. As decisões de sequenciamento estão relacionadas com a ordem de produção dos materiais e o dimensionamento de lotes está relacionado aos itens finais. O modelo matemático considera custos e tempos de preparação dependentes da sequência. Resultados dos testes computacionais realizados usando o pacote computacional Cplex 10.0 mostraram que o desempenho do método branch-and-cut pode ser melhorado com a reformulação a priori proposta.

Palavras-chave: programação inteira-mista; planejamento de produção; dimensionamento e sequenciamento de lotes; formulação estendida; reformulação a priori.

\begin{abstract}
In this paper, some new constraints and an extended formulation are presented for a Lot Sizing and Scheduling Model proposed in the literature. In the production process considered a key material is prepared and is transformed into different final items. The sequencing decisions are related to the order in which the materials are processed and the lot sizing decisions are related to the final items production. The mathematical formulation considers sequence-dependent setup costs and times. Results of the computational tests executed using the software Cplex 10.0 showed that the performance of the branch-and-cut method can be improved by the proposed a priori reformulation.
\end{abstract}

Keywords: mixed-integer programming; production planning; lot sizing and scheduling; extended formulation; a priori reformulation. 


\section{Introdução}

Em meio às decisões do planejamento da produção de algumas indústrias, uma questão frequente é como resolver o Problema de Dimensionamento de Lotes. Este problema consiste, basicamente, em determinar o tamanho dos lotes de produção de cada item a ser produzido em uma ou mais máquinas em cada período ao longo de um horizonte de planejamento finito. Os primeiros estudos de problemas de dimensionamento de lotes ocorreram com o Economic Order Quantity (EOQ) em 1913 (Harris, 1990) e, nas últimas décadas, vários modelos de otimização inteira mista foram propostos para o problema.

Jans \& Degraeve (2008) observam que as diferentes aplicações industriais têm feito surgir diferentes adaptações dos modelos clássicos de dimensionamento de lotes. Como exemplo, em alguns processos industriais tem-se a produção de mais de um item em um mesmo período e a ordem de produção desses itens é relevante. Neste caso, além do dimensionamento, as decisões a serem tomadas envolvem também o sequenciamento dos lotes, configurando assim um Problema de Dimensionamento e Sequenciamento de Lotes. Na prática, a maioria das empresas, bem como em vários trabalhos da literatura, as decisões sobre o tamanho dos lotes e a sequência em que deverão ser produzidos são consideradas de forma independente. Num primeiro nível são definidos quais itens serão produzidos a cada período (dimensionamento dos lotes) e, num segundo nível, a sequência de produção de tais itens é determinada. No entanto, o número de trabalhos na literatura que trata o problema de forma integrada vem crescendo bastante nos últimos anos. Algumas revisões bibliográficas podem ser encontradas em: Drexl \& Kimms (1997), Karimi et al. (2003) e Jans \& Degraeve (2007, 2008). Aplicações industriais recentes de modelos integrados de dimensionamento e sequenciamento aparecem, por exemplo, na produção de bebidas (Toledo et al., 2007 e Ferreira et al., 2008, 2009), ração (Toso et al., 2009), grãos eletrofundidos (Lucke et al., 2008), embalagens de vidro (Almada-Lobo et al., 2007, 2008) e fundição (Araujo et al., 2008).

Em alguns processos industriais, por exemplo, no setor de fundição e bebidas, as decisões de sequenciamento não estão relacionadas diretamente com os itens, mas sim, com a sequência de produção de determinados materiais que serão utilizados para produzir um grupo de itens demandados, que devem ser fabricados com aquele tipo de material produzido. Nestes casos, a integração dos dois níveis de decisão passa a ser ainda mais importante, pois, além da possibilidade de melhorar a sequência de produção dos materiais ainda é possível dimensionar grupos de itens que usam o mesmo material reduzindo as necessidades de trocas.

Dentre os trabalhos que consideram este tipo de situação tem-se Araujo et al. (2007) que fazem uma descrição genérica do problema e resolvem um modelo considerando custos e tempos de preparação dependentes da sequência. Diante da dificuldade de resolução do modelo proposto por pacotes computacionais de uso geral, são desenvolvidos métodos heurísticos baseados em uma estratégia relax-and-fix (Wolsey, 1998; Kelly \& Mann, 2004) e na técnica de horizonte rolante de planejamento. Em Araujo et al. (2004, 2008) são apresentados estudos sobre o processo de produção de uma indústria de pequeno porte do setor fundições. O planejamento de produção nesta indústria consiste em determinar a sequência de processamento de ligas de metal e o tamanho dos lotes de itens finais que são produzidos a partir de cada liga. O modelo apresentado em Araujo et al. (2008) trata o sequenciamento das ligas e o dimensionamento dos lotes de forma integrada considerando custos e tempos de preparação independentes da sequência. Em Araujo et al. (2004) os tempos de preparação são desconsiderados. 
Quando se propõe um modelo matemático para uma nova situação prática, a necessidade de construir modelos simplificados aliada às dificuldades oriundas do processo de modelagem (levantamento de dados, definição dos pontos principais a serem considerados e, principalmente, validação do modelo) pode fazer com que a qualidade do modelo proposto, em termos teóricos/computacionais (por exemplo, a qualidade da relaxação linear), não seja tratada de forma relevante. No presente trabalho é feita uma análise do efeito causado nos resultados computacionais quando algumas modificações são feitas na modelagem de um problema. Estudamos a reformulação do modelo proposto em Araujo et al. (2007). Uma das modificações proposta consiste numa formulação estendida do modelo original. Além disso, são propostas adições de novas restrições com o propósito de melhorar a qualidade do limitante inferior associado a relaxação linear do problema e assim melhorar o desempenho do algoritmo branch-and-cut incluído no sistema Cplex.

Na próxima seção (Seção 2) o modelo proposto em Araujo et al. (2007) é apresentado resumidamente. Na Seção 3 é apresentada uma formulação estendida e na Seção 4 são propostas algumas novas restrições. Na Seção 5 é apresentado um estudo computacional para avaliar a qualidade das reformulações propostas considerando o desempenho do método branch-and-cut incluído no pacote computacional Cplex 10.0. Na Seção 6 são apresentadas as conclusões do trabalho.

\section{Formulação Matemática}

Resumidamente, o processo produtivo estudado em Araujo et al. (2007) tem as seguintes características: um material pode ser usado na fabricação de vários produtos, mas um produto só pode ser feito com um único tipo de material. Além disso, um produto só pode ser fabricado em determinado período se o material que ele utiliza também tiver sido produzido neste período, pois não é permitido estoque de material de um período para outro. Apenas um tipo de material pode ser produzido de cada vez e a capacidade da máquina que produz os materiais deve ser respeitada, considerando que o tempo de preparação da máquina depende da sequência de produção dos materiais. São permitidos atrasos no atendimento à demanda.

As decisões de sequenciamento no modelo integrado proposto em Araujo et al. (2007) são obtidas dividindo-se um período em subperíodos menores, como é feito no modelo GLSP (General Lot Sizing and Scheduling Problem) proposto por Drexl \& Kimms (1997). Supondo conhecidos, $K$ o total de materiais, $P$ o total de itens, $T$ o total de períodos e $\eta$ o total de subperíodos, considere os seguintes índices usados no modelo:

$j, k=1, \cdots, K-$ materiais;

$p=1, \cdots, P-$ itens;

$t=1, \cdots, T-$ períodos;

$n=1, \cdots, \eta-$ subperíodos.

Para facilitar indexação na descrição do modelo, são considerados ainda:

$\eta_{t}$ - número de subperíodos do período $t$. Note que $\eta=\sum_{t=1}^{T} \eta_{t}$ é o número total de subperíodos.

Pesquisa Operacional, v.30, n.3, p.637-655, Setembro a Dezembro de 2010 
$F_{t}=1+\sum_{\tau=1}^{t-1} \eta_{\tau}-$ índice do primeiro subperíodo do período $t$. Note que $F_{1}=1$

$L_{t}=F_{t}+\eta_{t}-1-$ indica o índice do último subperíodo do período $t$. Note que $L_{T}=\sum_{t=1}^{T} \eta_{t}$.

Os seguintes parâmetros são considerados conhecidos:

$C$ : capacidade total disponível da máquina em cada subperíodo para o preparo da máquina e processamento do material;

$\rho_{p}$ : capacidade exigida da máquina para a produção do material necessário para produzir uma unidade do item $p$;

$d_{p t}:$ demanda do item $p$ no período $t$;

$S(k)$ : conjunto de itens $p$ que são produzidos a partir do material $k$. Note que $\{1, \cdots, P\}=S(1) \cup \cdots \cup S(K)$ e $S(k) \cap S(j)=\varnothing$ para todos os materiais $j$ e $k$ com $j \neq k$, já que cada item é produzido a partir de um único material;

$h_{p t}^{+}:$custo por estocar uma unidade do item $p$ no fim período $t$;

$h_{p t}^{-}$: custo por atrasar a produção de uma unidade do item $p$ no fim do período $t$;

$s_{j k}$ : custo de preparo da máquina pela troca do material $j$ para o material $k$. Considere

$s_{j j}=0$ para todo $j$ e que vale a desigualdade triangular, ou seja, $s_{i j} \leq s_{i k}+s_{k j}$ para todo $i$, $j$ e $k$;

$s t_{j k}$ : perda da capacidade da máquina se houver a troca do material $j$ para o material $k$. Supomos que $s t_{j j}=0$ para todo $j$ e que vale a desigualdade triangular, ou seja, $s t_{i j} \leq s t_{i k}+s t_{k j}$ para todo $i, j$ e $k$.

Considere as seguintes variáveis de decisão:

$x_{p n}$ : quantidade do item $p$ a ser produzida no subperíodo $n$;

$I_{p t}^{+}$: quantidade do item $p$ a ser estocada no fim do período $t$;

$I_{p t}^{-}:$quantidade em atraso do item $p$ no período $t ;$

$y_{n}^{k}$ : se for 1 , indica que a máquina deve ser preparada para processar o material $k$ no subperíodo $n$, e se for 0 , indica o caso contrário;

$z_{n}^{j k}:$ se for 1 , indica que deverá haver a troca do material $j$ para o material $k$ no subperíodo $n$, e se for 0 , indica o caso contrário. 
O modelo de otimização, que denotaremos por FO (Formulação Original), considerando um horizonte de planejamento com $T$ períodos é dado por:

Formulação Original (FO) (Araujo et al., 2007)

$$
\operatorname{Min} \sum_{p=1}^{P} \sum_{t=1}^{T}\left(h_{p t}^{-} I_{p t}^{-}+h_{p t}^{+} I_{p t}^{+}\right)+\sum_{j=1}^{K} \sum_{k=1}^{K} \sum_{n=F_{1}}^{L T} s_{j k} z_{n}^{j k}
$$

Sujeito a:

$$
\begin{array}{ll}
I_{p, t-1}^{+}-I_{p, t-1}^{-}+\sum_{n=F_{t}}^{L_{t}} x_{p n}-I_{p t}^{+}+I_{p t}^{-}=d_{p t} & \forall p, t \\
\sum_{p \in S(k)} \rho_{p} x_{p n}+s t_{j k} z_{n}^{j k} \leq C y_{n}^{k} & \forall j, k, n=F_{1}, \ldots, L_{T} \\
\sum_{k=1}^{K} y_{n}^{k}=1 & n=F_{1}, \ldots, L_{T} \\
z_{n}^{j k} \geq y_{n-1}^{j}+y_{n}^{k}-1 & \forall j, k, n=F_{1}, \ldots, L_{T} \\
\sum_{j=1}^{K} \sum_{k=1}^{K} z_{F_{t}}^{j k}=1 & \forall t \\
y_{n}^{k} \in\{0,1\}, y_{0}^{k}=0 & \forall k, n=F_{1}, \ldots, L_{T} \\
z_{n}^{j k} \geq 0 & \forall j, k, n=F_{1}, \ldots, L_{T} \\
I_{p t}^{+} \text {e } I_{p t}^{-} \geq 0 & \forall p, t \\
x_{p n} \geq 0 \text { e inteiro } & \forall p, n=F_{1}, \ldots, L_{T}
\end{array}
$$

A função objetivo (1) minimiza a soma dos custos gerados pela produção estocada e atrasada e pelas trocas efetuadas no sequenciamento dos preparos (no problema em questão vale a desigualdade triangular). $\mathrm{O}$ conjunto de restrições (2) determina o balanço entre o estoque, a produção em atraso e a produção total de um item $p$ em cada período $t$. As restrições (3) determinam que a quantidade de material processado em um subperíodo não deverá ultrapassar a capacidade da máquina e garantem que se um material $k$ for processado num subperíodo $n$, somente serão produzidos os itens que são feitos a partir dele. As restrições (4) asseguram que apenas um material seja processado por subperíodo.

As restrições (5) controlam a troca de materiais processados. Se no subperíodo $n-1$ a máquina foi preparada para a produção de um material $j\left(y_{n-1}^{j}=1\right)$ e no subperíodo $n$, para o material $k\left(y_{n}^{k}=1\right)$ a restrição (5) associada se torna $z_{n}^{j k} \geq 1$. Como o critério de otimização do problema é de minimização, a variável $z_{n}^{j k}$ recebe o valor 1 indicando a troca do material $j$ para o material $k$ no subperíodo $n$. Considerando-se a solução ótima, não é necessário definir $z_{n}^{j k}$ como variável binária, pois, como $y_{n}^{k}$ e $y_{n}^{j}$ são variáveis binárias, 
devido às restrições (7), as restrições (5) e o critério de otimização usado garantem que $z_{n}^{j k}$ sempre tomará o menor valor não negativo maior ou igual a $y_{n-1}^{j}+y_{n}^{k}-1$. Note que as restrições (7) fixam um valor nulo para as variáveis $y_{0}^{k}$ impondo que a máquina não está preparada para nenhum material no período zero. O conjunto de restrições (6) assegura a realização de uma troca no primeiro subperíodo de cada período. Embora essas restrições não estejam presentes diretamente na formulação apresentada em Araujo et al. (2007), os autores descrevem a presença das mesmas no texto (página 341). As restrições (8), (9) e (10) definem o domínio das variáveis. Para uma descrição detalhada do modelo e do processo produtivo representado veja Araujo et al. (2007).

\section{Reformulação Estendida}

Nesta seção, é desenvolvida uma formulação estendida (FE), para a formulação original (FO) descrita na Seção 2 baseada no conceito de redefinição de variável proposto por Eppen \& Martin (1987).

A formulação FE pode ser obtida interpretando as restrições (2) da formulação FO de maneira análoga às restrições de atendimento à demanda do Problema de Localização de Facilidades (Brahimi et al., 2006). Sejam $N=\{1, \cdots, \eta\}$ um conjunto de candidatos à instalação de facilidades e $M=\{1, \cdots, T\}$ um conjunto de clientes. O Problema de Localização de Facilidades consiste em determinar as facilidades que serão instaladas de forma que as demandas sejam satisfeitas com um custo mínimo. Para interpretar as restrições (2) da formulação FO como restrições de um problema de localização, supomos que a produção no subperíodo $n$ para satisfazer a demanda de um período $t$ equivale a utilizar uma facilidade $n$ instalada para satisfazer a demanda do cliente $t$. Assim, os subperíodos representam as facilidades, e a produção desses subperíodos será destinada a satisfazer a demanda de determinados períodos, que representam os clientes.

Para obter uma reformulação para a formulação FO, serão usadas as mesmas definições de índices, dados e variáveis da Seção 2. Adicionalmente, considere a seguinte variável:

$Q_{p n t}$ : indica a quantidade do item $p$ produzida no subperíodo $n$ para satisfazer a demanda no período $t$.

Dessa forma, a produção de um determinado subperíodo pode ter vários destinos como mostra a Figura 1. Considerando um horizonte de planejamento de 4 períodos com 3 subperíodos cada, a produção no subperíodo 6 pode ser usada para atender a demanda de qualquer subperíodo pertencente aos períodos 1,2, 3 ou 4 .

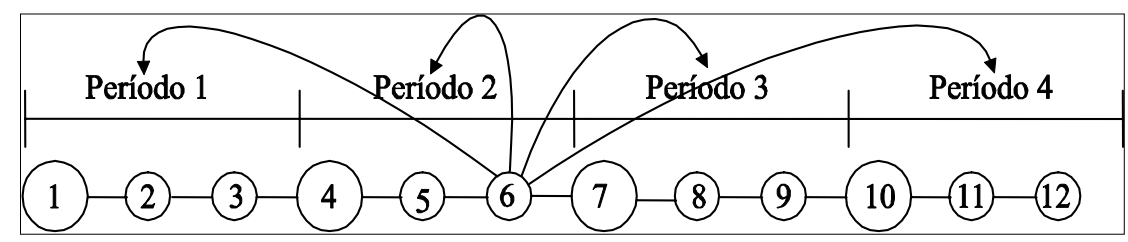

Figura 1 - Destinos possíveis da produção no subperíodo 6. 
Note que se a produção num subperíodo $n$ pertencente a um período $t$ for destinada ao período $t^{\prime}$ posterior a $t\left(t^{\prime}>t\right)$, então essa produção deverá ser estocada até o fim do período $\left(t^{\prime}-1\right)$, e por isso deverão ser contabilizados os custos de estoque nos períodos $t, \ldots, t^{\prime}-1$. Por outro lado, se a produção for destinada a um período $t^{\prime \prime}$ anterior a $t$, então essa é uma produção em atraso e por isso deverá também incluir os custos da produção em atraso nos períodos $t^{\prime \prime}, \ldots, t-1$.

Considerando que $\lambda_{n}$ indica o período ao qual um subperíodo $n$ pertence, os custos para o estoque $\left(C Q_{p n t}^{+}\right)$e para a produção em atraso $\left(C Q_{p n t}^{-}\right)$deverão ser redefinidos da seguinte maneira:

$C Q_{p n t}^{+}$: indica o custo de estocar uma unidade do produto $p$ no subperíodo $n$ para satisfazer a demanda no período $t$ posterior a $\lambda_{n}$. Note que $C Q_{p n t}^{+}=\sum_{l=\lambda_{n}}^{t-1} h_{p l}^{+}\left(\lambda_{n} \leq t\right)$.

$C Q_{p n t}^{-}$: indica o custo de atrasar uma unidade do produto $p$ no subperíodo $n$ para satisfazer a demanda no período $t$ anterior a $\lambda_{n}$. Note que $C Q_{p n t}^{-}=\sum_{l=t}^{\lambda_{n}-1} h_{p l}^{-}\left(\lambda_{n} \geq t\right)$.

A formulação estendida (FE) é então dada pela função objetivo (11), pelas novas restrições (12)-(14) definidas a seguir e as restrições (4)-(9) já definidas na formulação FO.

\section{Formulação Estendida (FE)}

$$
\operatorname{Min} \sum_{p=1}^{P}\left(\sum_{n=1}^{L_{T}-1} \sum_{t=\lambda_{n}+1}^{T} C Q_{p \lambda_{n} t}^{+} Q_{p n t}+\sum_{n=F_{2}}^{L_{T}+1} \sum_{t=1}^{\lambda_{n}-1} C Q_{p \lambda_{n}}^{-} Q_{p n t}\right)+\sum_{j=1}^{K} \sum_{k=1}^{K} \sum_{n=F_{1}}^{L_{T}} s_{j k} z_{n}^{j k}
$$

Sujeito à:

(4)-(9)

$$
\begin{array}{ll}
\sum_{n=F_{1}}^{L_{T}+1} Q_{p n t}=d_{p t} & \forall p, t \\
\sum_{p \in S(k)} \rho_{p}\left(\sum_{t=1}^{T} Q_{p n t}\right)+s t_{j k} z_{n}^{j k} \leq C y_{n}^{k} & \forall j, k, n=F_{1}, \ldots, L_{T} \\
Q_{p n t} \geq 0 \text { e inteiro } & \forall p, t, n=F_{1}, \ldots, L_{T}
\end{array}
$$

A função objetivo (11) minimiza a soma dos custos com a produção estocada, em atraso e com as trocas efetuadas. As restrições (12) garantem que a demanda de um período $t$ seja atendida pela produção originada de todos os possíveis subperíodos. Observe que para permitir a produção em atraso no último período, foi necessário definir a variável de produção $Q$ para $\eta+1$ subperíodos. Note também que o estoque inicial é nulo. As restrições (13) são idênticas às restrições (3) da FO, porém escritas com as novas variáveis de produção e, finalmente, as restrições (14) definem o domínio das variáveis. Embora se tenha um aumento no número de variáveis, alguns trabalhos da literatura aplicados a outros problemas 
de dimensionamento de lotes têm mostrado que esta estratégia de redefinição de variáveis (reformulação), em geral, melhora a qualidade dos limitantes obtidos do modelo. Veja Brahimi et al. (2006) e Jans \& Degraeve (2007, Seção 3.5) para algumas discussões recentes sobre trabalhos que utilizam tais estratégias.

Deve-se destacar que é possível escrever as variáveis de produção, estoque e de produção em atraso da formulação $\mathrm{FO}$ em função das variáveis de produção da formulação FE, ou $\quad$ seja, $\quad x_{p n}=\sum_{t=1}^{T} Q_{p n t} \quad \forall p=1, \ldots, P \quad n=F_{1}, \ldots, F_{T}, \quad I_{p t}^{+}=\sum_{n=F_{t}}^{L_{t}} \sum_{l=t+1}^{T} Q_{p n l} \quad \forall t=1, \ldots, T \quad \mathrm{e}$ $I_{p t}^{-}=\sum_{n=F_{t+1}}^{T+1} Q_{p n t} \quad \forall t=1, \ldots, T$, respectivamente.

\section{Adição de Restrições}

Nesta seção, são apresentadas algumas restrições que podem ser adicionadas às formulações FO e FE. Tais restrições foram geradas a partir de considerações associadas às variáveis de troca e preparo. Essas restrições são baseadas em alguns trabalhos da literatura: Koçlar (2005), Wolsey (2002), Belvaux \& Wolsey (2001).

\subsection{Equações de Fluxo (V1)}

$\mathrm{Na}$ relaxação linear das formulações $\mathrm{FO}$ e $\mathrm{FE}$, as variáveis de preparo e troca têm uma ligação fraca. As variáveis de preparo podem assumir valores positivos, permitindo a produção, sem que as variáveis de troca sejam necessariamente positivas. Por exemplo: se $y_{n-1}^{j}=0,6$ e $y_{n}^{k}=0,4$ então, de acordo com a restrição (5) tem-se que, $z_{n}^{j k} \geq 0,6+0,4-1=0$. Este tipo de solução implica em uma perda na qualidade do limitante gerado pela relaxação linear (utilizado no método branch-and-cut).

A família de equações V1 apresentadas a seguir, baseadas em Wolsey (2002), estabelecem uma ligação mais forte entre as variáveis de preparo e troca. Quando houver preparo do material $k$ em um subperíodo $n$ (por exemplo $y_{n}^{k}=0,4$ ), a troca de um determinado material para o material $k$ deve ser considerada (equação V1a, $\sum_{j=1}^{K} z_{n}^{j k}=0,4$ ); da mesma forma, quando o material $j$ estiver preparado em um subperíodo $n-1$ (por exemplo $y_{n-1}^{j}=0,6$ ), a troca do material $j$ para algum outro material (pode ser o próprio $j$ ) no subperíodo $n$ também deve ser considerada (equação $\mathrm{V} 1 \mathrm{~b}, \sum_{k=1}^{K} z_{n}^{j k}=0,6$ ).

\section{(V1)}

(V1a) $\sum_{j=1}^{K} z_{n}^{j k}=y_{n}^{k}, k=1, \cdots, K, n=F_{1}+1, \cdots, L_{T}$.
(V1b) $\sum_{k=1}^{K} z_{n}^{j k}=y_{n-1}^{j}, j=1, \cdots, K, n=F_{1}+2, \cdots, L_{T}$. 


\subsection{Inequações desagregadas de Fluxo (V2)}

$\mathrm{O}$ conjunto de inequações $\mathrm{V} 2$ permitem que uma variável de troca de um material $j$ para um material $k$ assuma valores maiores que zero somente quando as variáveis de preparo para o material $k$ no subperíodo $n$ e do material $j$ no subperíodo $n-1$ tiverem também valores maiores que zero. Além disso, o valor da variável de troca não pode ser maior que o valor da variável de preparo.

(V2)

c) $z_{n}^{j k} \leq y_{n}^{k}, j=1, \cdots, K, k=1, \cdots, K, n=F_{1}+1, \cdots, L_{T}$.

d) $z_{m}^{j k} \leq y_{n-1}^{j}, j=1, \cdots, K, k=1, \cdots, K, n=F_{1}+2, \cdots, L_{T}$.

\subsection{Inequações de Preparo e Trocas (V3)}

Constantino (1995) e Belvaux \& Wolsey (2001) descrevem um conjunto de inequações que, após uma adaptação para o problema tratado aqui, estabelecem três possibilidades mutuamente exclusivas: o material $i$ é preparado no subperíodo $n-1$; existe uma troca de um material $j$ para o item $i$ no subperíodo $n(j \neq i)$; ou algum material $j$ diferente de $i$ é preparado em ambos os subperíodos $n$ e $n-1$.

$$
y_{n-1}^{i}+\sum_{j=1 \mid j \neq i}^{K} z_{n}^{j i}+\sum_{j=1 \mid j \neq i}^{K}\left(y_{n}^{j}-\sum_{k=1 \mid k \neq j}^{K} z_{n}^{k j}\right) \leq 1, i=1, \cdots, K, n=F_{1}, \cdots, L_{T} .
$$

Em (V3) são consideradas apenas as trocas de materiais diferentes para subperíodos sucessivos. Note que se $y_{n-1}^{i}$ for 1 , então necessariamente $\sum_{j=1 \mid j \neq i}^{K} z_{n}^{j i}$ será igual a $0 \mathrm{e}$, se $y_{n-1}^{i}$ for 0 , então $\sum_{j=1 \mid j \neq i}^{K} z_{n}^{j i}$ poderá ser igual a 0 ou 1 . Assim, quando $y_{n-1}^{i}$ for 1 , tem-se que: $\sum_{j=1 \mid j \neq i}^{K} y_{n}^{j} \leq \sum_{j=1 \mid j \neq i}^{K} \sum_{k=1 \mid k \neq j}^{K} z_{n}^{k j}$.

Neste caso, as inequações V3 evitam que as variáveis de preparo assumam valores maiores que zero quando as trocas forem nulas. O mesmo ocorre quando $y_{n-1}^{i}$ for 0 e $\sum_{j=1 j j i}^{K} z_{n}^{j i}$ for 1. Quando $y_{n-1}^{i}$ for 0 e $\sum_{j=1 \mid j \neq i}^{K} z_{n}^{j i}$ também for 0 não há nenhuma implicação sobre as outras variáveis.

\subsection{Inequações de Troca para $i=j(\mathrm{~V} 4)$}

As inequações V4 estabelecem uma relação mais forte do que as restrições de troca (5) entre as variáveis de preparo e troca quando $i=j$. Elas generalizam as inequações definidas para problemas small bucket que aparecem em Belvaux \& Wolsey (2001) que permitem produção de no máximo dois itens por períodos.

$$
y_{n-1}^{j}+y_{n}^{j}+\sum_{k=1 k \neq j}^{K} z_{n}^{k k} \leq 1+z_{n}^{j j}, \forall j=1, \cdots, K, n=F_{1}, \cdots, L_{T} .
$$


A análise destas inequações pode ser feita considerando 3 casos diferentes: se houver preparo para o item $j$ em subperíodos consecutivos $\left(y_{n-1}^{j}=y_{n}^{j}=1\right.$ ), então $\sum_{k=1 \mid k \neq j}^{K} z_{n}^{k k}$ é igual a zero e $z_{n}^{j j}$ é forçada a receber o valor 1 , indicando a troca do material $j$ para ele mesmo no subperíodo $n$; se $y_{n-1}^{j}+y_{n}^{j}=1$, ou seja, o preparo para o item $j$ ocorrerá no subperíodo $n-1$ ou no subperíodo $n$, então itens diferentes serão produzidos em subperíodos consecutivos e, portanto $z_{n}^{i j}$ e $\sum_{k=1 \mid k \neq j}^{K} z_{n}^{k k}$ serão iguais a zero, isto é, deverá ocorrer a troca de um material $k(k \neq j)$ para o material $j$ ou a troca do material $j$ para um material $k(j \neq k)$; se $y_{n-1}^{j}+y_{n}^{j}=0$, ou seja, não há preparo para o item $j$ nos subperíodos $n$ e $n-1$, então $z_{n}^{j j}$ é igual a zero. Portanto, a inequação resulta em $\sum_{k=1 \mid k \neq j}^{K} z_{n}^{k k} \leq 1$, pois somente uma troca por subperíodo pode ocorrer.

\subsection{Inequações de Eliminação de Trocas Não-Necessárias (E1)}

Suponha que uma solução para o problema tenha em um dos seus períodos uma sequência na qual o mesmo material seja processado duas vezes em dois subperíodos não consecutivos. Observe que uma solução melhor (ou igual) pode ser obtida redefinindo a sequência de produção fazendo com que esse material seja processado em subperíodos consecutivos. As redefinições das sequências não influenciam na capacidade disponível, já que a desigualdade triangular é igualmente válida para os tempos de preparo com as trocas efetuadas.

Diante dessa análise, pode-se afirmar que existe uma solução ótima tal que, se, num mesmo período, houver a necessidade de mais de um preparo para um mesmo material, os preparos devem ocorrer em subperíodos consecutivos. As inequações E1 impõem esta restrição:

$$
\sum_{j=1 \mid j \neq k}^{K} \sum_{n=F_{t}}^{L_{t}} z_{n}^{j k} \leq 1, \forall k=1, \cdots, K, t=1, \cdots, T .
$$

As inequações E1 não permitem que a troca de um material $j$ para um material $k$, dado que $j$ seja diferente de $k$, ocorra mais de uma vez no mesmo período. Esta classe de inequações não é válida para o problema uma vez que elimina soluções factíveis, porém a sua inclusão na formulação não elimina a solução ótima.

Maiores detalhes sobre as equações V1 e inequações V2, V3, V4 e E1 podem ser encontrados em Bernardes (2008) e nas referências citadas.

\section{Testes Computacionais}

Foram realizados testes computacionais com as duas formulações (FO) e (FE) apresentadas na Seção 2 e na Seção 3, respectivamente. Os testes foram feitos sem a adição (Pura) e com a adição a priori das restrições descritas na Seção 4 de forma individual e combinada. $O$ objetivo é analisar o desempenho do método branch-and-cut na solução de exemplares destas formulações e verificar se este desempenho pode ser melhorado com a adição a priori 
de restrições. Os modelos foram codificados na sintaxe do AMPL (Fourer et al., 1993) e resolvidos pelo pacote computacional Cplex 10.0 com parâmetros default, utilizando um computador AMD Athlon $(2.81 \mathrm{GHz})$ com 1.87 de memória RAM, sob a plataforma Window XP.

Os resultados mostrados nas Tabelas 2, 3,4 e 5 consideram a média dos melhores limites inferiores (LI), melhores limites superiores (LS), dos gaps, dos números de nós do branchand-cut (Nós) e dos números de planos de corte gerados pelo Cplex (PC). Deve-se ressaltar que o gap exibido é fornecido pelo pacote Cplex e, de acordo com ILOG 2006 (página 270) é calculado da seguinte forma: $g a p=|L S-L I| /\left(e^{-10}+|L S|\right)$.

\subsection{Geração de Dados}

Foram testados exemplares com 10 itens e 2 materiais, 50 itens e 10 materiais e 100 itens e 20 materiais, configurando três tamanhos (pequeno, médio e grande, respectivamente). Para cada um dos tamanhos, são consideradas combinações de três variações da capacidade (folgada, normal ou apertada) e duas variações do custo de preparo (alto ou baixo), totalizando em 18 tipos de problemas. Os dados foram gerados aleatoriamente e são os mesmos de Araujo et al. (2007). A Tabela 1 apresenta resumidamente os parâmetros usados. $\mathrm{O}$ cálculo das capacidades (CAP) é feito da seguinte forma: $\mathrm{CAP}=\left(\sum_{p=1}^{P} \sum_{t=1}^{T} d_{p t} \rho_{p}\right) / L_{T}$. O horizonte de planejamento é igual para todos os exemplares, 5 períodos divididos em 10 subperíodos cada um.

Tabela 1 - Representação da Geração de Dados.

\begin{tabular}{ll}
\hline \hline Parâmetros & Definições \\
\hline & Problema Pequeno: $(10,2)$ \\
Número de itens e materiais & Problema Médio: $(50,10)$ \\
\hline Número de períodos $(T)$ & Problema Grande: $(100,20)$ \\
\hline Custo de estoque $\left(h_{p t}^{+}\right)$ & 5 \\
\hline Custo de atraso $\left(h_{p t}^{-}\right)$ & {$[2,10]$} \\
\hline Capacidade para a produção de uma unidade do item $p\left(\rho_{p}\right)$ & {$[20,100]$} \\
\hline Tempo de preparo $\left(s t_{j k}\right)$ & {$[0,1,3]$} \\
\hline Custo de preparo $\left(s_{j k}\right)$ & {$[5,10]$} \\
\hline Demanda $\left(d_{p t}\right)$ & baixo: $50 \times s t_{j k}$ \\
\hline & alto: $500 \times s t_{j k}$ \\
\hline Capacidade $(C)$ & {$[40,60]$} \\
\hline \hline
\end{tabular}




\subsection{Testes Preliminares}

Testes preliminares foram realizados considerando exemplares com 10, 50, 100 itens e a combinação capacidade normal e baixo custo de preparação. Escolheu-se esta combinação porque ela representa exemplares intermediários em termos de dificuldade de resolução. $\mathrm{O}$ objetivo foi identificar a combinação: formulação e família de restrições, com efeito mais positivo no desempenho do método branch-and-cut incluído no Cplex. Foram resolvidos 5 exemplares para cada tamanho de problema com o tempo limite de uma hora cada. Os parâmetros analisados resultam de uma média de 5 valores. No caso dos problemas com 10 itens, para os quais a solução ótima é obtida dentro do tempo limite, observou-se uma pequena redução do tempo em que a solução ótima é encontrada quando as famílias V1, V3, V4 e E1 foram adicionadas na formulação FO. Considerando a formulação FE, o tempo de resolução foi reduzido somente com a adição da família V4 e, com a adição simultânea das famílias V1 e E1. Para os problemas com 100 itens, não foi possível obter soluções factíveis, dentro do limite de tempo estabelecido, para vários exemplares. Como a análise é baseada em apenas 5 exemplares, não foi possível obter conclusões consistentes com relação a adição das restrições propostas para este conjunto de problemas.

A seguir, na Tabela 2 são apresentados com maiores detalhes os testes preliminares para os problemas com 50 itens. O tempo de resolução foi limitado em 1 hora. Além das formulações puras (sem adição das restrições propostas), são mostrados também os resultados de cada restrição adicionada individualmente, bem como, da adição simultânea das restrições V1 e E1 (V1+E1), que foi a que obteve os melhores resultados entre as combinações testadas.

Tabela 2 - Testes preliminares com os exemplares de 50 itens.

\begin{tabular}{l|l|lllll} 
Restrições & Formulação & LS & LI & gap (\%) & Nós & PC \\
\hline \hline Pura & FO & 51.564 & 4.846 & 90,49 & 5.751 & 4.656 \\
& FE & 32.971 & 1.456 & 95,38 & 24.350 & 2.992 \\
\hline V1 & FO & 33.700 & 2.723 & 92,04 & 12.255 & 2.463 \\
& FE & 32.516 & 10.054 & 69,08 & 12.714 & 2.848 \\
\hline V2 & FO & 40.820 & 4.746 & 88,33 & 3.363 & 2.464 \\
& FE & 49.940 & 75 & 100,00 & 13.398 & 2.933 \\
\hline V3 & FO & 41.123 & 2.104 & 94,86 & 5.291 & 4.552 \\
& FE & 35.707 & 8.649 & 75,86 & 22.023 & 2.941 \\
\hline V4 & FO & 48.222 & 4.969 & 89,58 & 5.126 & 4.552 \\
& FE & 34.636 & 296 & 99,09 & 22.023 & 2.941 \\
\hline E1 & FO & 67.691 & 4.753 & 91,67 & 4.023 & 4.118 \\
& FE & 35.496 & 587 & 97,98 & 23.115 & 2.989 \\
\hline V1+E1 & FO & 33.403 & 3.018 & 90,97 & 6.587 & 3.043 \\
& FE & 31.452 & 10.072 & 67,90 & 10.997 & 2.881 \\
\hline
\end{tabular}

A adição do conjunto de restrições $\mathrm{V} 1$ e $\mathrm{V} 1+\mathrm{E} 1$ teve um efeito bastante positivo na formulação FE, provocando uma expressiva redução do gap. Nos casos em que foram adicionadas as famílias V2, V4 e E1, o efeito não foi positivo. Para todos os casos envolvendo a formulação $\mathrm{FE}$, não houve variação significativa do número de planos de corte gerados. As restrições V1 e V2 causam uma grande redução no número de nós examinados no limite de tempo. 
Para a formulação FO, observou-se uma melhoria expressiva no limitante superior com a inclusão das restrições propostas na Seção 4, com exceção para a família E1 e destaque para as famílias V1 e V1+E1. O melhor limite inferior foi obtido com as restrições V4, no entanto o menor gap foi obtido com a combinação $\mathrm{FO}+\mathrm{V} 2$. As restrições $\mathrm{V} 1$ causam uma grande redução no número de planos de corte gerados e um aumento no número de nós. Mais detalhes sobre os testes preliminares pode ser encontrado em Bernardes (2008).

A partir dos resultados dos testes preliminares foram realizados novos testes computacionais com a formulação FO proposta em Araujo et al. (2007) e a formulação FE+V1+E1 que apresentou o melhor desempenho considerando o gap associado às formulações testadas. Os resultados dos novos testes são descritos na próxima seção.

\subsection{Resultados Computacionais}

As Tabelas 3, 4 e 5 apresentam os resultados médios de 10 exemplares para cada um dos 18 tipos de problemas que surgem a partir da combinação de parâmetros da Tabela 1. As formulações $\mathrm{FO}$ e FE+V1+E1 foram resolvidas com o tempo de resolução limitado em 1 hora. Para problemas com 100 itens, o tempo limite de resolução foi de 2 horas, tendo em vista a dificuldade de encontrar as soluções viáveis no tempo de 1 hora.

Analisando na Tabela 3 os resultados para os problemas com 10 itens tem-se que: ambas as formulações obtiveram bons resultados encontrando a solução ótima para todos os exemplares de duas combinações de parâmetros diferentes. Considerando o gap médio a formulação $\mathrm{FE}+\mathrm{V} 1+\mathrm{E} 1$ obtém resultados suavemente melhores $1,15 \%$ contra $1,18 \%$ da formulação FO. No entanto, considerando individualmente cada uma das seis combinações de parâmetros, a formulação $\mathrm{FO}$ obtém resultados melhores em quatro combinações. A formulação $\mathrm{FE}+\mathrm{V} 1+\mathrm{E} 1$ utiliza maior número de planos de corte enquanto FO utiliza maior número de nós.

Tabela 3 - Testes com os exemplares de 10 itens.

\begin{tabular}{|c|c|c|c|c|c|c|}
\hline \multicolumn{2}{|c|}{ Problema } & \multicolumn{5}{|c|}{ Formulação FO (Pura) } \\
\hline Capacidade & Custo de preparo & LS & LI & $\operatorname{gap}(\%)$ & Nós & $\mathrm{PC}$ \\
\hline \multirow[t]{2}{*}{ relaxada } & baixo & 1.961 & 1.961 & $0,00(4,59) *$ & 6.536 & 118 \\
\hline & alto & 13.883 & 12.961 & 0,68 & 2.605 .419 & 109 \\
\hline \multirow[t]{2}{*}{ normal } & baixo & 1.961 & 1.961 & $0,00(2,72)^{*}$ & 2.221 & 132 \\
\hline & alto & 14.405 & 14.382 & 0,16 & 7.656 .824 & 149 \\
\hline \multirow[t]{2}{*}{ apertada } & baixo & 6.340 & 6.023 & 5,22 & 7.212 .994 & 194 \\
\hline & alto & 22.576 & 22.388 & 1,04 & 7.723 .037 & 184 \\
\hline \multicolumn{2}{|c|}{ Média } & 10.187 & 9.946 & 1,18 & 4.201 .170 & 148 \\
\hline \multicolumn{2}{|c|}{ Problema } & \multicolumn{5}{|c|}{ Formulação FE + V1 + E1 } \\
\hline Capacidade & Custo de preparo & $\mathrm{LS}$ & LI & $\operatorname{gap}(\%)$ & Nós & $\mathrm{PC}$ \\
\hline \multirow[t]{2}{*}{ relaxada } & baixo & 1.961 & 1.961 & $0,00(20,37)^{*}$ & 5.919 & 469 \\
\hline & alto & 13.040 & 13.033 & 0,05 & 836.071 & 512 \\
\hline \multirow[t]{2}{*}{ normal } & baixo & 1.961 & 1.961 & $0,00(10,20)^{*}$ & 3.392 & 365 \\
\hline & alto & 14.416 & 14.373 & 0,28 & 4.852 .745 & 141 \\
\hline \multirow[t]{2}{*}{ apertada } & baixo & 6.366 & 6.019 & 5,45 & 1.425 .233 & 382 \\
\hline & alto & 22.622 & 22.362 & 1,12 & 1.933 .979 & 353 \\
\hline \multicolumn{2}{|c|}{ Média } & 10.061 & 9.952 & 1,15 & 1.509 .556 & 370 \\
\hline
\end{tabular}

* O valor entre parênteses mostra o tempo médio em segundos gastos para resolver os 10 exemplares 
No caso dos problemas com 50 itens (Tabela 4), observa-se que, quando utiliza-se a formulação $\mathrm{FE}+\mathrm{V} 1+\mathrm{E} 1$ há uma sensível melhora nas soluções viáveis (Limitantes Superiores (LS)) e uma expressiva melhora nos limitantes inferiores (LI) ocasionando uma redução no gap médio de $90,1 \%$ para $74,35 \%$. O número de planos de corte é semelhante e a formulação $\mathrm{FE}+\mathrm{V} 1+\mathrm{E} 1$ explora um número de nós menor no limite de tempo.

Tabela 4 - Testes com os exemplares de 50 itens.

\begin{tabular}{|c|c|c|c|c|c|c|}
\hline \multicolumn{2}{|c|}{ Problema } & \multicolumn{5}{|c|}{ Formulação FO (Pura) } \\
\hline Capacidade & Custo de preparo & LS & LI & $\operatorname{gap}(\%)$ & Nós & $\mathrm{PC}$ \\
\hline \multirow[t]{2}{*}{ relaxada } & baixo & 20.574 & 113 & 99,41 & 107.798 & 609 \\
\hline & alto & 118.504 & 495 & 99 & 79.880 & 618 \\
\hline \multirow[t]{2}{*}{ normal } & baixo & 46.277 & 4.555 & 89,11 & 13.413 & 4.305 \\
\hline & alto & 189.900 & 5.983 & 96,88 & 9.581 & 4.406 \\
\hline \multirow[t]{2}{*}{ apertada } & baixo & 113.906 & 33.898 & 69,82 & 5.778 & 4.878 \\
\hline & alto & 258.274 & 35.071 & 86,39 & 5.243 & 4.910 \\
\hline \multicolumn{2}{|c|}{ Média } & 124.572 & 13.352 & 90,1 & 36.949 & 3.287 \\
\hline \multicolumn{2}{|c|}{ Problema } & \multicolumn{5}{|c|}{ Formulação FE + V1 + E1 } \\
\hline Capacidade & Custo de preparo & LS & LI & $\operatorname{gap}(\%)$ & Nós & $\mathrm{PC}$ \\
\hline \multirow[t]{2}{*}{ relaxada } & baixo & 19.924 & 3.088 & 84,69 & 21.405 & 2.899 \\
\hline & alto & 119.115 & 9.157 & 92,25 & 13.706 & 3.016 \\
\hline \multirow[t]{2}{*}{ Normal } & baixo & 30.588 & 10.223 & 66,43 & 11.311 & 2.869 \\
\hline & alto & 133.498 & 22.923 & 82,74 & 5.434 & 3.064 \\
\hline \multirow[t]{2}{*}{ Apertada } & baixo & 93.944 & 44.605 & 52,44 & 5.319 & 3.231 \\
\hline & alto & 200.860 & 65.125 & 67,54 & 4.012 & 3.357 \\
\hline \multicolumn{2}{|c|}{ Média } & 99.655 & 25.853 & 74,35 & 10.198 & 3.073 \\
\hline
\end{tabular}

Considerando os problemas com 100 itens (Tabela 5), não foram obtidas soluções factíveis para 22 dos 60 exemplares da formulação $\mathrm{FE}+\mathrm{V} 1+\mathrm{E} 1$, enquanto a formulação $\mathrm{FO}$ obteve soluções factíveis para todos os 60 exemplares. No entanto, para os exemplares em que foram obtidas soluções factíveis a formulação $\mathrm{FE}+\mathrm{V} 1+\mathrm{E} 1$ obteve resultados positivos melhorando os limites superiores e limites inferiores. O gap médio da formulação $\mathrm{FE}+\mathrm{V} 1+\mathrm{E} 1$, considerando apenas os exemplares em que foram obtidas soluções factíveis, foi de $51,90 \%$ enquanto se consideramos apenas esses exemplares o gap médio da formulação FO é $88,48 \%$.

Por se tratar de uma formulação com maior número de variáveis o algoritmo branch-and-cut usado pelo Cplex apresentou maior dificuldade na resolução desses exemplares. Esta dificuldade pode ser solucionada considerando-se outros métodos para a solução desta formulação no caso de exemplares de grande porte, por exemplo, o uso de geração de colunas e/ou heurísticas, um tópico interessante para trabalhos futuros. 
Tabela 5 - Testes com os exemplares de 100 itens.

\begin{tabular}{|c|c|c|c|c|c|c|}
\hline \multicolumn{2}{|c|}{ Problema } & \multicolumn{5}{|c|}{ Formulação FO (Pura) } \\
\hline Capacidade & Custo de preparo & LS & LI & $\operatorname{gap}(\%)$ & Nós & $\mathrm{PC}$ \\
\hline \multirow[t]{2}{*}{ relaxada } & baixo & 3.168 .824 & 34.289 & 96,39 & 407 & 2.733 \\
\hline & alto & 4.013 .845 & 35.373 & 99,12 & 192 & 2.620 \\
\hline \multirow[t]{2}{*}{ normal } & baixo & 1.724 .305 & 62.179 & 87,46 & 763 & 3.021 \\
\hline & alto & 3.024 .839 & 62.108 & 94,61 & 735 & 2.755 \\
\hline \multirow[t]{2}{*}{ apertada } & baixo & 425.144 & 219.685 & 47,96 & 2.862 & 217.057 \\
\hline & alto & 613.137 & 217.057 & 64,44 & 2.167 & 3.485 \\
\hline \multicolumn{2}{|c|}{ Média } & 2.161 .682 & 105.115 & 81,66 & 1.188 & 38.612 \\
\hline \multicolumn{2}{|c|}{ Problema } & \multicolumn{5}{|c|}{ Formulação FE + V1 + E1 } \\
\hline Capacidade & Custo de preparo & LS & LI & $\operatorname{gap}(\%)$ & Nós & $\mathrm{PC}$ \\
\hline \multirow[t]{2}{*}{ relaxada } & baixo & 132.762 & 91.869 & 30,77 & 2.894 & 7345 \\
\hline & alto & $293.448 *$ & 96.072 & 63,17 & 2.493 & 7488 \\
\hline \multirow[t]{2}{*}{ normal } & baixo & $275.127 * *$ & 97.227 & 63,95 & 1.009 & 6573 \\
\hline & alto & 399.695 & 130.613 & 67,15 & 1.481 & 7409 \\
\hline \multirow[t]{2}{*}{ apertada } & baixo & ---- & 196.689 & ---- & 248 & 2153 \\
\hline & alto & $530.846 * * *$ & 283.110 & 50,64 & 502 & 3761 \\
\hline \multicolumn{2}{|c|}{ Média } & 326.376 & 149.263 & 55,14 & 1.438 & 5788 \\
\hline
\end{tabular}

* Indica que somente para 9 exemplares entre os 10 foram encontradas soluções viáveis.

** Indica que somente para 7 exemplares entre os 10 foram encontradas soluções viáveis.

*** Indica que somente para 2 exemplares entre os 10 foram encontradas soluções viáveis.

---- Indica que não foram encontradas soluções viáveis para os exemplares dentro tempo limite.

\subsection{Comparação com Procedimentos Heurísticos}

Em Araujo et al. (2007) os exemplares da formulação FO pura foram resolvidos com o pacote computacional Cplex 9.0 e também utilizando as heurísticas Descent Heuristic (DH), Diminishing Neighbourhood Search (DN) e Simulated Annealing (SA).

O objetivo desta seção é avaliar os resultados da formulação proposta, FE+V1+E1, comparando com os resultados das heurísticas DH, DN e SA.

Inicialmente, para avaliar o efeito da versão 10.0 do pacote Cplex (usada no presente artigo) na qualidade dos resultados computacionais, comparamos na Tabela 6 a formulação FO pura e as heurísticas desenvolvidas em Araujo et al. (2007). A formulação FO pura foi resolvida com o pacote Cplex 10.0 no tempo limite de uma hora. Os dados na Tabela 6 consistem na diferença em porcentagem entre as soluções das heurísticas e da formulação FO, ou seja:

$$
\text { Diferença }=\frac{(\text { Solução da heurística }- \text { Solução do CPLEX) }}{\text { Solução do CPLEX }} \times 100 \% \text {. }
$$


Tabela 6 - Diferença (\%) entre a solução da formulação FO pura e as das Heurísticas de Araujo et al. (2007).

\begin{tabular}{cc|ccccccc}
\hline \hline \multicolumn{2}{c}{ Método } & \multicolumn{2}{c}{ DH } & \multicolumn{2}{c}{ DN } & \multicolumn{2}{c}{ AS } \\
\hline \hline \multirow{2}{*}{ Problema } & & & & \multicolumn{2}{c}{ Custo de Preparo } \\
\hline \multirow{3}{*}{10 itens } & Capacidade & baixo & alto & baixo & alto & baixo & alto \\
& relaxada & 25,82 & 56,35 & 6,34 & 50,27 & 6,34 & 50,26 \\
& normal & 90,73 & 60,25 & 12,89 & 44,80 & 6,34 & 44,81 \\
& apertada & 61,47 & 32,36 & $-20,11$ & 4,57 & $-20,97$ & 7,94 \\
\hline \multirow{3}{*}{50 itens } & relaxada & 33,67 & 36,56 & 11,49 & 16,13 & 13,86 & 16,49 \\
& normal & $-6,51$ & $-10,85$ & $-14,58$ & $-24,60$ & $-15,40$ & $-23,72$ \\
& apertada & 5,32 & $-12,17$ & $-3,78$ & $-19,27$ & 1,57 & $-20,40$ \\
\hline \multirow{3}{*}{100 itens } & relaxada & $-92,48$ & $-90,65$ & $-92,55$ & $-91,19$ & $-92,61$ & $-91,17$ \\
& normal & $-79,41$ & $-83,63$ & $-79,84$ & $-84,50$ & $-80,19$ & $-84,52$ \\
& apertada & 34,56 & 9,06 & 29,02 & 1,55 & 27,54 & 5,28 \\
\hline \hline
\end{tabular}

Para exemplos com 50 e 100 itens as heurísticas fornecem soluções melhores que as soluções encontradas pela resolução da formulação FO pura para a maior parte dos problemas. Para os problemas com 50 itens e capacidade relaxada e com 100 itens e capacidade apertada, a formulação FO obteve resultados melhores do que todas as heurísticas. Para problemas pequenos (10 itens) a resolução da formulação FO obtém resultados melhores, o que já era esperado devido aos resultados semelhantes obtidos em Araujo et al. (2007).

Na Tabela 7 apresentam-se as diferenças entre as soluções fornecidas pelas heurísticas DH, $\mathrm{DN}$ e SA com relação às soluções encontradas ao se resolver o modelo FE+V1+E1 com o pacote Cplex 10.0 no tempo limite de uma hora, para os exemplares com 10 e 50 itens, e de duas horas para os exemplares com 100 itens.

Tabela 7 - Diferença (\%) entre a solução da formulação FE+V1+E1 e das Heurísticas de Araujo et al. (2007).

\begin{tabular}{cc|cccccc}
\hline \multicolumn{2}{c}{ Método } & \multicolumn{2}{c}{ DH } & \multicolumn{2}{c}{ DN } & \multicolumn{2}{c}{ AS } \\
\hline \hline \multirow{2}{*}{ Problema } & & & & \multicolumn{2}{c}{ Custo de Preparo } \\
\hline \multirow{3}{*}{10 itens } & Capacidade & baixo & alto & baixo & alto & baixo & alto \\
& relaxada & 25,82 & 66,46 & 6,34 & 59,99 & 6,34 & 59,97 \\
& normal & 90,73 & 60,12 & 12,89 & 44,69 & 6,34 & 44,70 \\
& apertada & 60,81 & 32,09 & $-20,44$ & 4,36 & $-21,29$ & 7,72 \\
\hline \multirow{3}{*}{50 itens } & relaxada & 38,04 & 35,86 & 15,12 & 15,53 & 17,57 & 15,90 \\
& normal & 41,30 & 26,81 & 29,09 & 7,25 & 27,86 & 8,51 \\
& apertada & 27,70 & 12,94 & 16,66 & 3,81 & 23,15 & 2,35 \\
\hline \multirow{2}{*}{100 itens } & relaxada & 79,38 & $27,95 *$ & 77,85 & $20,49 *$ & 76,38 & $20,73 *$ \\
& normal & $29,02 * *$ & $23,86 *$ & $26,32 * *$ & $17,34 *$ & $24,17 * *$ & $17,12^{*}$ \\
& apertada & ---- & 25,96 & ---- & 17,29 & ----- & 21,60 \\
\hline \hline
\end{tabular}

\footnotetext{
* Indica que somente para 6 exemplares entre os 10 foram encontradas soluções viáveis pelo Cplex.

** Indica que somente para 2 exemplares entre os 10 foram encontradas soluções viáveis pelo Cplex.

----Indica que não foram encontradas soluções viáveis para os exemplares dentro tempo limite do Cplex.
} 
Note que, para os problemas com 10 itens, as soluções fornecidas pelas heurísticas continuam piores na maioria dos casos. Considerando os problemas com 50 itens, ao contrário do que aconteceu na Tabela 6 , as soluções da encontradas pela resolução da $\mathrm{FE}+\mathrm{V} 1+\mathrm{E} 1$ foram entre $3 \%$ e $41 \%$ melhores que as soluções das heurísticas. Com relação aos problemas com 100 itens, novamente a formulação FE+V1+E1 apresenta dificuldades em encontrar uma solução factível para alguns exemplares. Foram calculadas as diferenças apenas para os casos em que o Cplex 10.0 encontrou alguma solução factível dentro do tempo limite e, para estes casos analisados, em média as heurísticas fornecem soluções entre $17 \%$ e $79 \%$ piores.

Em geral, considerando a média das 3 heurísticas para todos os exemplares, de acordo com os resultados da Tabela 6, tem-se que as soluções heurísticas são 7,99\% melhores do que a as soluções encontradas pela resolução da formulação FO com o pacote Cplex 10.0. Por outro lado, considerando a Tabela 7, onde se tem a resolução da FE+V1+E1 com o mesmo pacote, as heurísticas ficam $27,93 \%$ piores. Portanto, considerando os exemplares em que foi possível encontrar uma solução factível, os procedimentos propostos neste artigo conseguiram melhorar substancialmente os resultados obtidos pelas heurísticas desenvolvidas em Araujo et al. (2007).

\section{Conclusão}

Neste trabalho, estudou-se um Problema de Dimensionamento e Sequenciamento de Lotes, para um processo produtivo em que é necessária a produção de um material bruto que será transformado, posteriormente, em itens finais. Os custos e perdas de capacidade com preparo são considerados dependentes da sequência.

Diante da dificuldade de resolução do problema com um pacote computacional, em Araujo et al. (2007) foram desenvolvidas algumas heurísticas e o método relax-and-fix foi usado para resolver o problema num horizonte rolante de planejamento. No presente trabalho foi desenvolvida uma formulação estendida e foram propostas novas restrições com o objetivo de obter uma formulação mais forte para o modelo proposto em Araujo et al. (2007).

Os resultados computacionais mostraram que algumas inequações propostas não tiveram grandes efeitos sobre a qualidade da solução enquanto outras tiveram efeitos bastante positivos. Em geral, verificou-se que as restrições V1+E1 adicionadas a priori na formulação estendida (FE) melhoraram a qualidade dos limitantes obtidos e, com esta reformulação foi possível obter soluções melhores, considerando os exemplares para os quais foram obtidas soluções factíveis, do que aquelas obtidas pelas heurísticas desenvolvidas em Araujo et al. (2007).

Como trabalhos futuros, têm-se os estudos de outras reformulações, como, por exemplo, a inclusão de outras inequações válidas no modelo para melhorar ainda mais a formulação atual e a inclusão a posteriori das restrições propostas. Os procedimentos heurísticos propostos em Araujo et al. (2007) podem ser especializados à nova formulação proposta. $\mathrm{O}$ uso de procedimentos de geração de colunas e a extensão do problema para o caso em que se têm várias máquinas para a produção do "material bruto" também são tópicos interessantes para serem estudados.

Pesquisa Operacional, v.30, n.3, p.637-655, Setembro a Dezembro de 2010 


\section{Agradecimentos}

Os autores agradecem as contribuições feitas pelos árbitros anônimos da revista Pesquisa Operacional, que muito melhoraram esta versão do trabalho e o apoio da Fundação de Amparo à Pesquisa do Estado de São Paulo (FAPESP) e do Conselho Nacional de Desenvolvimento Científico e Tecnológico (CNPq).

\section{Referências Bibliográficas}

(1) Anily, S.; Tzur, M. \& Wolsey, L.A. (2008). Multi-item lot sizing with joint setup costs. Mathematical Programming, 119(1), 79-94.

(2) Araujo, S.A. \& Arenales, M.N. (2004). Planejamento e programação da produção numa fundição cativa automatizada de grande porte. Investigação Operacional, 24, 197-210.

(3) Araujo, S.A.; Arenales, M.N. \& Clark, A.R. (2007). Joint rolling-horizon scheduling of materials processing and lot sizing with sequence dependent. Journal of Heuristics, 13, 337-358.

(4) Araujo, S.A.; Arenales, M.N. \& Clark, A.R. (2008). Lot sizing and furnace scheduling in small foundries. Computers \& Operations Research, 35, 916-932.

(5) Belvaux G. \& Wolsey, L.A. (2001). Modelling pratical lot sizing problems as mixedinteger programs. Management Science, 47(7), 993-1007.

(6) Bernardes, E.D. (2008). Reformulação e inequações válidas para um problema integrado de dimensionamento de lotes. Dissertação de Mestrado, Ibilce/Unesp, São José do Rio Preto, SP.

(7) Brahimi, N.; Dauzere-Peres, S.; Najid, N.M. \& Nordli, A. (2006). Single Item Lot Sizing Problems. European Journal of Operational Research, 168, 1-16.

(8) Almada Lobo, B.; Oliveira, J.F. \& Carravilla, M.A. (2008). Production planning and scheduling in the glass container industry: A VNS approach. International Journal of Production Economics, 114(1), 363-375.

(9) Almada Lobo, B.; Klabjan, D.; Carravilla, M.A. \& Oliveira, J.F. (2007). Single machine multi-product capacitated lot sizing with sequence-dependent setups. International Journal of Production Research, 45(20), 4873-4894.

(10) Brahimi, N.; Dauzere-Peres, S.; Najid, N.M. \& Nordli, A. (2006). Single Item Lot Sizing Problems. European Journal of Operational Research, 168, 1-16.

(11) Constantino, M. (1995). A polyhedral approach to production planning models: start-up costs and times, upper and lower bounds on production. Ph.D. Dissertation, Universit'e Catholique de Louvain, Belgium.

(12) Drexl, A. \& Kimms, A. (1997). Lot sizing and scheduling - survey and extensions. European Journal of Operational Research, 99, 221-235.

(13) Ferreira, D.; Morabito, R. \& Rangel, S. (2008). Um modelo de otimização inteira mista e heurísticas relax and fix para a programação da produção de fábricas de refrigerantes de pequeno porte. Produção, 18, 76-88. 
(14) Ferreira, D.; Morabito, R. \& Rangel, S. (2009). Solution approaches for the soft drink integrated production lot sizing and scheduling problem. European Journal of Operational Research, 196, 697-706.

(15) Fleischmann, B. \& Meyr, H. (1997). The general lotsizing and scheduling problem. Operational Research Spektrum, 19, 11-21.

(16) Fourer, R.; Gay, D.M. \& Kernighan, B.W. (1993). AMPL- A Modeling Language for Mathematical Programming. Boyd and Frase, Danvers, Massachusetts.

(17) Harris, F.W. (1990). How many parts to make at once. Operations Research, 38, 947-950 (reprint from Factory-The Magazine of Management 10 (1913) 135-136, 152).

(18) ILOG (2006). ILOG Cplex 10: User’s Manual.

(19) Jans, R. \& Degraeve, Z. (2007). Meta-Heuristics for Dynamic Lot Sizing: A Review and Comparison of Solution Approaches. European Journal of Operational Research, 177, $1855-1875$.

(20) Jans, R. \& Degraeve, Z. (2008). Modeling Industrial Lot Sizing Problems: A Review. International Journal of Production Research, 46(6), 1619-1643.

(21) Kelly, J.D. \& Mann, J.L. (2004). Flowsheet Decomposition Heuristic For Scheduling: a Relax-and-Fix Method. Computers and Chemical Engineering, 28, 2193-2200.

(22) Koçlar, A. (2005). The general lot sizing and scheduling problem with sequence dependent changeovers. Dissertação de Mestrado, The Graduate School of Natural and Applied Sciences - Middle East Technical University, Ancara - Turquia.

(23) Luche, J.R.; Morabito, R. \& Pureza, V. (2008). Combining process selection and lot sizing models for the production scheduling of electrofused grains. Aceito para publicação no Asia-Pacific Journal of Operational Research.

(24) Meyr, H. (2000). Simultaneous Lotsizing and Scheduling by Combining Local Search with Dual Reoptimization. European Journal of Operational Research, 120, 311-326.

(25) Meyr, H. (2002). Simultaneous Lotsizing and Scheduling on Parallel Machines. European Journal of Operational Research, 139(2), 277-292.

(26) Toledo, C.F.; França, P.M.; Morabito, R. \& Kimms, A. (2007). Um modelo de otimização para o problema integrado de dimensionamento de lotes e programação da produção em fábricas de refrigerantes. Pesquisa Operacional, 27(1), 155-186.

(27) Toledo, C.F.; França, P.M.; Morabito, R. \& Kimms, A. (2008). A multi-population genetic algorithm to solve the synchronized and integrated two-level lot-sizing and scheduling problem, aceito para publicação no International Journal of Production Research. DOI: 10.1080/00207540701675833.

(28) Toso, E.; Morabito, R. \& Clark, A. (2009). Lot-sizing and sequencing optimisation at an animal-feed plant. Computers and Industrial Engineering, DOI: 10.1016/j.cie.2009.02.011, 2009.

(29) Wolsey, L.A. (1998). Integer Programming. Wiley, New York.

(30) Wolsey, L.A. (2002). Solving multi-item lot-sizing problems with an MIP solver using classification and reformulation. Management Science, 48(12), 1587-1602. 